\title{
Interacting random Dirac fermions in superconducting cuprates
}

\author{
D.V. Khveshchenko ${ }^{1}$, A.G. Yashenkin ${ }^{2,4, \dagger}$, and I.V.Gornyi ${ }^{3,4, \dagger \dagger}$ \\ ${ }^{1}$ Department of Physics and Astronomy, University of North Carolina, Chapel Hill, NC 27599 \\ ${ }^{2}$ Department of Physics, University of Florida, Gainesville, FL 32611 \\ ${ }^{3}$ Institut fur Nanotechnologie, Forschungszentrum Karlsruhe, 76021 Karlsruhe, Germany \\ ${ }^{4}$ NORDITA, Blegdamsvej 17, Copenhagen 2100, Denmark
}

\begin{abstract}
We study the effects of quasiparticle interactions on disorder-induced localization of Dirac-like nodal excitations in superconducting high- $T_{c}$ cuprates. As suggested by the experimental ARPES and terahertz conductivity data in $\mathrm{Bi}_{2} \mathrm{Sr}_{2} \mathrm{CaCu}_{2} \mathrm{O}_{8+\delta}$, we focus on the interactions mediated by the order parameter fluctuations near an incipient second pairing transition $d \rightarrow d+i s$. We find interaction corrections to the density of states, specific heat, and conductivity as well as phase and energy relaxation rates and assess the applicability of the recent localization scenarios for noninteracting random Dirac fermions to the cuprates.
\end{abstract}

In the past few years, the problem of disordered twodimensional (2D) Dirac fermions received much of attention, as it provides an effective description for the random bond Ising model, network models of Quantum Hall plateau transitions, and some other statistical problems. Also, dynamical Dirac fermions can be used to conveniently describe low-energy excitations in a variety of correlated systems, such as p-wave (e.g., $\mathrm{Ru}_{2} \mathrm{SrO}_{4}$ ) and layered d-wave (high- $T_{c}$ cuprates) superconductors and superfluids $\left(\mathrm{He}^{3}\right)$, and zero-gap semiconductors (e.g., graphene sheets).

The Hamiltonian of a generic disordered gapless superconductor and, in particular, planar $d$-wave system, possesses an additional discrete symmetry of charge conjugation which gives rise to as many as seven novel random Gaussian ensembles corresponding to different patterns of spin rotational and time reversal symmetry breaking [1]. Moreover, in a stark contrast with the conventional case of a normal metal with extended Fermi surface the density of states (DOS) of the non-interacting Dirac fermions in a $2 \mathrm{D}$ d-wave superconductor is strongly affected by disorder [2]. Furthermore, depending on the concrete model for disorder, such as isotropic versus predominantly forward potential impurity scattering, the DOS of the random Dirac fermions can exhibit different low-energy asymptotic behaviors even within the same random ensemble [3] 8 .

The multitude of different regimes and crossovers predicted for the non-interacting random Dirac fermions raises a question about their observability in such realistic $d$-wave systems as the high- $T_{c}$ superconductors where quasiparticle interactions are believed to be important. Moreover, the localization theory still remains incomplete without an extra input in the form of quasiparticle dephasing rate which controls the magnitude of disorder-induced localization corrections in the infinite system.

Thus far, despite the continuing progress in understaning of the non-interacting (de)localization phenomena in the $d$-wave systems, the above issues did not receive enough attention. In the present paper, we fill in this gap by investigating the effects of physically relevant quasiparticle interactions on the localization properties of the Dirac-like nodal excitations in the superconducting cuprates.

In the Nambu spinor representation, the quasiparticle (retarded) Green function reads as

$$
\hat{G}_{\mathbf{k}}^{R}(\epsilon)=\left[\left(\epsilon-\Sigma_{\mathbf{k}}^{R}(\epsilon)\right) \hat{\tau_{0}}+\xi_{\mathbf{k}} \hat{\tau_{3}}+\Delta_{\mathbf{k}} \hat{\tau}_{1}\right]^{-1}
$$

where we introduced the $2 \times 2$ unity matrix $\hat{\tau}_{0}$ in addition to the triplet of the Pauli matrices $\hat{\tau}_{1,2,3}$.

The bare quasiparticle spectrum which is composed of the normal state dispersion $\xi_{\mathrm{k}}$ and the $d_{x^{2}-y^{2-}}$ symmetrical gap $\Delta_{\mathbf{k}}$ becomes linear $E_{\mathbf{k}}=\left[\xi_{\mathbf{k}}^{2}+\Delta_{\mathbf{k}}^{2}\right]^{1 / 2} \approx$ $\left[\left(\mathbf{v}_{f} \delta \mathbf{k}\right)^{2}+\left(\mathbf{v}_{g} \delta \mathbf{k}\right)^{2}\right]^{1 / 2}$ for momenta near each of the four gap nodes located at $\pm \mathbf{K}_{1,2}= \pm\left( \pm k_{F}, k_{F}\right) / \sqrt{2}$. The pairs of orthogonal vectors $\mathbf{v}_{f}=\left(\partial \xi_{\mathbf{k}} / \partial \mathbf{k}\right)$ and $\mathbf{v}_{g}=\left(\partial \Delta_{\mathbf{k}} / \partial \mathbf{k}\right)$ correspond to the components of the quasiparticle group velocity which are normal and tangent to the fudicial Fermi surface, respectively.

By using the standard DOS definition

$$
\nu(\epsilon)=-\frac{1}{\pi} \operatorname{Tr} \sum_{\mathbf{k}} \operatorname{Im} G_{\mathbf{k}}^{R}(\epsilon)
$$

and linearizing the spectrum near the nodes, one readily recovers the linear DOS $\nu(\epsilon)=|\epsilon| /\left(\pi v_{f} v_{g}\right)$ of the nodal low-energy excitations in a clean $2 \mathrm{D}$ d-wave superconductor.

The origin of the quasiparticle interactions in the cuprates remains a subject of an ongoing debate. The recent ARPES 10] and optical (terahertz) conductivity 11] data in $\mathrm{Bi}_{2} \mathrm{Sr}_{2} \mathrm{CaCu}_{2} \mathrm{O}_{8+\delta}$ seem to rule out any shortranged coupling that would have resulted in a $T^{3}$ temperature dependence of the inverse inelastic quasiparticle lifetime 12. Instead, these data show an approximately linear behavior which is suggestive of the possibility of some sort of a quantum-critical behavior 13].

In the common scenario of a quantum-critical point (QCP), the long-ranged interactions between the quasiparticles result from their exchange by fluctuations of the new order parameter. The recent analysis of different incipient orderings singled out one of the second pairing 
transitions $d_{x^{2}-y^{2}} \rightarrow d_{x^{2}-y^{2}}+i s\left(i d_{x y}^{\prime}\right)$ as the most plausible candidate 13. Alternatively, one can also consider the long-ranged interaction due to charge fluctuations in the vicinity of a crystal structural instability 14]. Yet another type of interaction mediated by the exchange of antiferromagnetic fluctuations with momenta $\mathbf{q} \approx \mathbf{Q}$ where $\mathbf{Q}=( \pm \pi, \pi)$ has been studied extensively in the context of the normal state of the cuprates and recently extended to the superconducting regime [15], albeit with no disorder included.

In order to put all of the above quasiparticle interactions into one unifying framework we consider a generic form of the bosonic collective mode propagator

$$
\hat{V}_{i}^{R}(\omega, \mathbf{q})=\frac{V_{i} \hat{\tau}_{i} \otimes \hat{\tau}_{i}}{(\omega / c)^{2}-(\mathbf{Q}-\mathbf{q})^{2}-\lambda^{2}-V_{i} \Pi_{i}^{R}(\omega, \mathbf{q})}
$$

which describes the interactions in the spin, pairing, and charge channels for $i=0,2$, and 3, respectively. In Eq.(3) the parameter $\lambda^{2}$ controls the proximity to the QCP, and the fermion polarization functions $\Pi_{i}^{R}(\omega, \mathbf{q})$ account for the feedback effect of the nodal Dirac fermions on the spectrum of the collective mode.

As regards modeling the disorder, we consider the experimentally relevant case of time-reversal and spinrotational invariant scattering due to non-magnetic impurities (class CI in the classification chartered in Ref. [1]) which we treat as scatterers with density $n_{i}$ coupled to the nodal Dirac fermions via the vertex $U_{\mathbf{k}, \mathbf{k}^{\prime}} \hat{\tau}_{3}$. The latter accounts for arbirary strength and momentum dependence of disorder scattering and gives rise to the scattering $T$-matrix

$$
\hat{T}_{\mathbf{k}, \mathbf{k}^{\prime}}(\epsilon)=\sum_{\mathbf{k}^{\prime \prime}}\left[\delta_{\mathbf{k}, \mathbf{k}^{\prime \prime}}-U_{\mathbf{k}, \mathbf{k}^{\prime \prime}} \hat{\tau}_{3} \hat{G}_{\mathbf{k}^{\prime \prime}}^{R}(\epsilon)\right]^{-1} U_{\mathbf{k}^{\prime \prime}, \mathbf{k}^{\prime}} \hat{\tau}_{3}
$$

where the first and second terms in the brackets correspond to the Gaussian and Unitary limits, respectively [2]. In the earlier studies employing non-abelian bosonization and nonlinear $\sigma$-model [3, 5,7] the latter regime describing strong impurities has remained unattainable, and a markedly different behavior predicted in this case [6,8 could not be readily addressed.

The first insight into the random Dirac problem can be obtained from the self-consistent equation derived in the standard non-crossed diagrammatic approximation [2]

$$
\hat{\Sigma}_{\mathbf{k}}^{R}(\epsilon)=n_{i} \sum_{\mathbf{k}^{\prime}} \hat{T}_{\mathbf{k}, \mathbf{k}^{\prime}}(\epsilon) \hat{G}_{\mathbf{k}^{\prime}}^{R}(\epsilon) \hat{T}_{\mathbf{k}^{\prime}, \mathbf{k}}(-\epsilon)
$$

As shown below, our neglecting the crossed diagrams is well justified by the large anisotropy of the Dirac spectrum $\left(v_{F} / v_{g} \sim 20\right)$ observed in the cuprates [9].

Provided that $n_{i}\left|\hat{T}_{\mathbf{k}, \mathbf{k}^{\prime}}(\epsilon)\right| / v_{f} v_{g}<<1$, for all the relevant energies and momenta, the solution of Eq.(5) manifests the emergence of a new energy scale $-\operatorname{Im} \Sigma^{R}(0)=$ $\gamma<<\Delta$ which separates between the ballistic and diffusive regimes and gives rise to the finite DOS

$$
\nu_{0}=\frac{2 \gamma}{\pi^{2} v_{f} v_{g}} \ln \frac{\Delta}{\gamma}
$$

In the non-crossed approximation, the spin and thermal conductivities which obey the Wiedemann-Franz law appear to be independent of disorder $\left(\hbar=k_{B}=1\right)$ :

$$
\sigma_{s}=\frac{3 \kappa}{4 \pi^{2} T}=\frac{1}{4 \pi^{2}} \frac{v_{f}^{2}+v_{g}^{2}}{v_{f} v_{g}}
$$

while the charge conductivity receives non-universal vertex corrections [2]. This is consistent with the fact that, unlike the charge of quasiparticles, their spin and energy are conserved, and, therefore, in the absence of localization they can both propagate diffusively [4].

In the diffusive $(\epsilon<\gamma)$ regime, the mean field DOS (6) and the "universal" conductivities (7) alike become subject to further corrections which stem from both disorderinduced weak localization and interference between disorder scattering and quasiparticle interactions. In order to compute these corrections one needs explicit expressions for all the gapless diffusion $(\mathcal{D})$ and Cooperon $(\mathcal{C})$ modes of the random Dirac problem beyond the ergodic limit studied in Ref. [1].

The abovementioned strong anisotropy of the Dirac spectrum in the cuprates implies $\sigma_{s} \gg 1$ which, apart from justifying the use of Eq.5 for calculating the fermion self-energy, allows one to resort to the standard ladder approximation for the propagators $\hat{\mathcal{D}}(\hat{\mathcal{C}})=$ $\sum_{i j} \mathcal{D}_{i j}\left(\mathcal{C}_{i j}\right) \hat{\tau}_{i} \otimes \hat{\tau}_{j}$ expanded in the basis of the tensor products of the $\hat{\tau}_{i}$-matrices.

By analogy with a normal metal, we first consider the ladders formed by one retarded and another advanced Green functions (RA-ladder) and find the following singular contributions to the propagators of the soft modes

$$
\mathcal{D}_{i j}\left(\mathcal{C}_{i j}\right)\left(\epsilon, \epsilon^{\prime}, \mathbf{q}\right)=\delta_{i j} \frac{\gamma^{2}}{\pi \nu_{0}} \frac{\eta_{i}^{D(C)}}{D q^{2}-i\left(\epsilon-\epsilon^{\prime}\right)}
$$

with the amplitudes $\eta_{i}^{D}=(1,1,1,1)$ and $\eta_{i}^{C}=$ $(1,1,-1,1)$ corresponding to the $\mathcal{D}$ and $\mathcal{C}$ propagators, respectively 16 .

As previously pointed out [4], the diffusion coefficient $D$ appearing in Eq.(8) must satisfy the Einstein relation $\sigma_{s}=D \nu_{0} / 4$. In our approach, this happens naturally, once the real part of the fermion self-energy $\operatorname{Re} \hat{\Sigma}_{\mathbf{k}}^{R}(\omega)$ from Eq. (5) is accounted for in the ladder equations.

As opposed to the case of a normal metal, in a superconductor the gapless poles also appear in the RR (AA)ladders due to the combination of processes of impurity scattering and Andreev reflection [1]. The corresponding propagators $\overline{\mathcal{D}}(\overline{\mathcal{C}})$ are related to (8) by virtue of the charge conjugation performed on one of the two lines of the ladder:

$$
\overline{\mathcal{D}}(\overline{\mathcal{C}})\left(\epsilon, \epsilon^{\prime}, \mathbf{q}\right)=-\sum_{i} \mathcal{C}_{i j}\left(\mathcal{D}_{i j}\right)\left(\epsilon,-\epsilon^{\prime}, \mathbf{q}\right)\left(\hat{\tau}_{2} \hat{\tau}_{i}^{*} \hat{\tau}_{2}\right) \otimes \hat{\tau}_{i}
$$


which can be cast in the form of Eq.(8) with $\bar{\eta}_{i}^{D}=$ $(-1,1,-1,1)$ and $\bar{\eta}_{i}^{C}=(-1,1,1,1)$.

A straightforward analysis shows that it is the RRCooperon $\overline{\mathcal{C}}$ which appears to be solely responsible for the first order weak localization DOS correction [5]

$$
\delta_{w l} \nu(\epsilon)=-\frac{1}{\pi^{2} D} \ln \frac{\gamma}{\epsilon}
$$

Eq.(10) indicates that perturbation expansion remains well under control for energies above the characteristic scale $\sim \gamma \exp \left(-4 \pi^{2} \sigma_{s}\right)$ where it eventually breaks down.

Besides the above soft modes, in the case of a nearly nested (square-like) normal state Fermi surface there might also exist additional (pseudo)Goldstone ones with gaps of the order of chemical potential $\mu$ of the bare electrons. In the $d$-wave superconducting state, the corresponding poles occur in both RA- and RR(AA)-ladders when the sum of the two momenta carried by the lines equals $\mathbf{Q}$, provided that the normal state dispersion satisfies $\xi_{\mathbf{k}} \approx-\xi_{\mathbf{k} \pm \mathbf{Q}}$. In the Unitary limit, the presence of these extra modes reverses the sign of the localization correction (10) in the energy interval $\mu^{2} / \gamma<\epsilon<\gamma$ [16], resulting in a behavior which is reminiscent of that predicted in Refs. [6, 8] for $\mu=0$. Nonetheless, at yet lower energies the DOS will eventually get suppressed, in accord with the scenario of Refs. 叫5].

Next, we calculate the interaction (Altshuler-Aronov type) corrections which stem from the interplay between disorder and quasiparticle interactions (3). As usual, the latter can be divided into the exchange and Hartree contributions.

Upon computing the diffusion-dressed interaction vertices, we find that away from half-filling $\left(2 \mathbf{K}_{1,2} \neq \mathbf{Q}\right)$ no diffusion poles occur for any finite transferred momenta $\mathbf{Q} \neq 0$ which limits the subsequent discussion to the intra-node inelastic scattering.

Furthermore, the vertices $\hat{\tau}_{1,3}$ undergo no singular diffusion dressing, as they correspond to the two spatial components of the Dirac fermion current operator. By contrast, both vertices $\hat{\tau}_{0}$ and $\hat{\tau}_{2}$ which represent the Dirac fermion density and mass operators do develop diffusion poles in the RA- and RR(AA)-channels, respectively.

In light of the above, from now on we focus on the coupling $\hat{V}_{2}^{R}(\omega, \mathbf{q})$ mediated by the fluctuations of a secondary pairing order parameter with $\mathbf{Q}=0$ which was suggested as a possible scattering mechanism in the high temperature (ballistic) regime 13]. Here we restrict our attention to the case of the is secondary pairing, for the case of the $i d_{x y}$ pairing appears to be somewhat more intricate [17]. Unlike any other before mentioned types of interactions, it is strongly enhanced by disorder, thus producing the diffusive DOS correction

$$
\begin{aligned}
& \frac{\delta_{e x} \nu(\epsilon)}{\nu_{0}}= \\
& \int_{-\infty}^{\infty} \frac{d \omega}{2 \pi} \sum_{\mathbf{q}}\left[-\frac{1}{2} \tanh \frac{\epsilon-\omega}{2 T} \operatorname{Im} \frac{V_{2}^{R}(\omega, \mathbf{q})}{\left(D q^{2}-i(2 \epsilon-\omega)\right)^{2}}\right. \\
& \left.\quad+\operatorname{coth} \frac{\omega}{2 T} \operatorname{Im}_{2}^{R}(\omega, \mathbf{q}) \operatorname{Re} \frac{1}{\left(D q^{2}-i(2 \epsilon-\omega)\right)^{2}}\right]
\end{aligned}
$$

In the conserving approximation, the above vertex renormalization is necessarily accompanied by the singular polarization of the disordered Dirac fermions

$$
\Pi_{2}^{R}(\omega, \mathbf{q})=\nu_{0} \ln \frac{\gamma}{D q^{2}-i \omega}
$$

Close to the QCP $\left(\lambda^{2}<V_{2} \nu_{0}\right)$, the term (12) dominates over the other terms in the denominator of Eq.(3), thereby resulting in the intrinsically attractive coupling $\hat{V}_{2}^{R}(\omega, \mathbf{q}) \approx-1 / \Pi_{2}^{R}(\omega, \mathbf{q})$ which yields a positive DOS correction

$$
\delta_{e x} \nu(\epsilon)=\frac{1}{4 \pi^{2} D} \ln \left|\ln \frac{\gamma}{\epsilon}\right|
$$

Thus, in contrast to the exchange DOS correction in a normal metal, in our case $\delta \nu_{e x}$ appears to be substantially weaker than the effect of weak localization (10).

Farther away from the QCP $\left(\lambda^{2}>V_{2} \nu_{0}\right)$, the bosonic propagator (3) becomes effectively short-ranged, and the (positive) interaction correction to DOS becomes explicitly dependent upon the coupling strength: $\delta_{e x} \nu(\epsilon) \sim$ $\left(V_{2} \nu_{0} / \lambda^{2}\right) \ln \gamma / \epsilon$, while the corresponding Hartree term vanishes identically.

The latter regime appears to be partly similar to the case of a short-ranged non-singular ferromagnetic coupling of the local quasiparticle spin densities in a gapless bulk superconductor which yields the interaction DOS correction of the same functional form and sign as the localization one [18].

The DOS correction (13) can also be deduced from the singular contributions to thermodynamic quantities, e.g., specific heat

$$
\begin{gathered}
\delta C(T)=T \frac{d^{2}}{d T^{2}} \int_{0}^{\infty} \frac{d \omega}{2 \pi}\left[\operatorname{coth}\left(\frac{\omega}{2 T}\right)+1\right] \\
\sum_{\mathbf{q}} \tan ^{-1} \frac{\operatorname{Im} \Pi_{2}^{R}(\omega, \mathbf{q})}{\operatorname{Re} \Pi_{2}^{R}(\omega, q)}=\frac{T}{2 \pi^{2} D} \ln \left|\ln \frac{\gamma}{T}\right|
\end{gathered}
$$

calculated for $\lambda \rightarrow 0$. Accordingly, the exchange contribution to the conductivity correction is given by the expression

$$
\begin{array}{r}
\delta_{e x} \sigma_{s}(T)=-2 \sigma_{s} \operatorname{Im} \int_{-\infty}^{\infty} \frac{d \omega}{2 \pi} \frac{d}{d \omega}\left[\omega \operatorname{coth}\left(\frac{\omega}{2 T}\right)\right] \\
\sum_{\mathbf{q}} \frac{D q^{2} V_{2}^{R}(\omega, \mathbf{q})}{\left[D q^{2}-i \omega\right]^{3}}=\frac{1}{8 \pi^{2}} \ln \left|\ln \frac{\gamma}{T}\right|
\end{array}
$$


which also shows a much weaker temperature dependence than the logarithmic weak-localization correction $\delta_{w l} \sigma_{s}(T)=-\ln \left(\gamma \tau_{\phi}(T)\right) / 2 \pi^{2}$ controlled by the inelastic phase-breaking time $\tau_{\phi}(T)$ 任.

The latter can be estimated as a time interval for which the accumulated phase uncertainty

$$
\begin{array}{r}
\delta \Phi(t)=\int_{-\infty}^{\infty} \frac{d \omega}{2 \pi} \operatorname{coth}\left(\frac{\omega}{2 T}\right) \\
\sum_{\mathbf{q}} \operatorname{Im} V_{2}^{R}(\omega, \mathbf{q}) \frac{1-\exp \left(i \omega t-D q^{2} t\right)}{\left[D q^{2}-i \omega\right]^{2}}
\end{array}
$$

becomes of order unity [20]. From the condition $\delta \Phi\left(\tau_{\phi}\right) \sim$ 1 , we obtain the estimate

$$
\tau_{\phi}^{-1}(T) \sim \frac{T}{16 \pi \sigma_{s} \ln ^{2} \gamma / T}
$$

that should be contrasted against the apparent phasebreaking rate $\propto T^{1 / 3}$ found to describe both, experimentally and theoretically, quasiparticle transport in the normal state of the cuprates 21].

Also, the dephasing time (17) differs from the inelastic energy relaxation (or, "out-scattering") time [19]

$$
\begin{array}{r}
\tau_{\epsilon}^{-1}=\int_{-\infty}^{\infty} \frac{d \omega}{2 \pi}\left[\operatorname{coth}\left(\frac{\omega}{2 T}\right)-\tanh \left(\frac{\omega}{2 T}\right)\right] \\
\sum_{\mathbf{q}} \operatorname{Im} V_{2}^{R}(\omega, \mathbf{q}) \frac{1}{D q^{2}-i \omega+\tau_{\epsilon}^{-1}}
\end{array}
$$

For $T<<\gamma / \sigma_{s}$ the solution of this self-consistent equation can be found in the form

$$
\tau_{\epsilon}^{-1}(T)=\frac{T \ln \sigma_{s}}{16 \pi \sigma_{s} \ln ^{2} \gamma / T}
$$

Unlike $\tau_{\phi}$ which is determined by the processes with energy transters $\omega \sim \tau_{\phi}^{-1}, \tau_{\epsilon}$ receives contributions from the frequencies up to $\omega \sim T$. In the ballistic $(T>\gamma)$ regime, $\tau_{\epsilon}^{-1}(T)$ becomes a linear function of temperature, in agreement with the earlier theoretical [13] and experimental 10,11] results.

In conclusion, we carried out the analysis of the effects of the experimentally relevant quasiparticle interactions mediated by fluctuations of a secondary pairing order parameter $(i s)$ on the localization properties of the nodal quasiparticles in disordered high- $T_{c}$ superconducors. In the course of this study we revisited the problem of localization of noninteracting random Dirac fermions and explicitly solved for all the relevant diffusion and Cooperon modes in a $2 \mathrm{D}$ d-wave superconductor with arbitrarily strong impurities. We found that, despite being singular as a function of transferred energy and momentum, the interaction in question generates only subdominant, albeit positive, DOS, specific heat, and conductivity corrections and, therefore, does not necessarily alter the main predictions of the non-interacting localization scenario of Refs. [4, [, [7]. Lastly, we provided the so far missing ingredient of the localization theory by computing the inelastic dephasing and energy relaxation.

We note, in passing, that the latter does not account for the possibility of the formation of a strongly anisotropic network of delocalized resonant impurity states, as proposed in Ref. 222]. However, as far as the thermodynamic and transport properties of the rest of the electronic spectrum are concerned, our findings provide further support for the attempts to find manifestations of the quasiparticle localization-related phenomena in the superconducting cuprates [23].

Meanwhile, the high precision heat transport measurements [24,25] might have already provided such an important evidence of weak localization as positive magneto(thermal)conductivity. Should this interpretation of the data of Ref. [25] proposed in [23] prove to be correct, the phase relaxation time (17) may as well turn out to be experimentally accessible.

The authors are grateful to A. Altland for his valuable comments on the case of the $i d$ secondary pairing and also on Eq.(11). This research was supported by the NSF under Grants DMR-0071362 (DVK) and DMR-9703388 (AGY), by RFBR (IVG), and partly by INTAS.

$\dagger \quad$ Also at Petersburg Nuclear Physics Institute, 188300 St. Petersburg, Russia.

†† Also at A.F. Ioffe Physico-Technical Institute, 194021 St. Petersburg, Russia.

[1] A.Altland and M.R.Zirnbauer, Phys.Rev.B55, 1142 (1997).

[2] P.A.Lee, Phys.Rev.Lett.71, 1887 (1993); A.Durst and P.A.Lee, Phys.Rev.B62, 1270 (2000).

[3] A.A. Nersesyan, A.M. Tsvelik, and F. Wenger, Phys. Rev. Lett. 72, 2628 (1994); Nucl. Phys. B438, 561 (1995).

[4] T. Senthil, M.P.A. Fisher, L. Balents, and C. Nayak, Phys. Rev. Lett. 81, 4704 (1998).

[5] T. Senthil and M.P.A. Fisher, Phys. Rev. B 60, 6893 (1999).

[6] C. Pepin and P.A. Lee, Phys. Rev. B63, 054502 (2001).

[7] A. Altland, B.D. Simons, and M.R. Zirnbauer, condmat/0006362.

[8] C. Chamon and C. Mudry, Phys. Rev. B63, 100503 (2001).

[9] J. Mesot et al, Phys. Rev. Lett. 83, 840 (1999); M. Chiao et al, Phys. Rev. B62, 3554 (2000).

[10] T. Valla et al., Science 285, 2110 (1999); Phys. Rev. Lett. $\mathbf{8 5}, 828$ (2000).

[11] J. Corson et al., Phys. Rev. Lett. 85, 2569 (2000).

[12] S.M. Quinlan, D.J. Scalapino, N. Bulut, Phys. Rev. B49, 1470 (1994). 
[13] M. Vojta, Y.Zhang, and S.Sachdev, Phys. Rev. B62, 6721 (2000); D.V.Khveshchenko and J.Paaske, Phys. Rev. Lett. 86, 4672 (2001).

[14] M.Grilli, DiCastro, and C.Castellani, Phys.Rev.Lett.75, 4650 (1995).

[15] Ar.Abanov and A.V.Chubukov, Phys. Rev. Lett. 83, 1652 (1999).

[16] A.G. Yashenkin, W.A. Atkinson, I.V. Gornyi, P.J. Hirschfeld, and D.V. Khveshchenko, Phys. Rev. Lett. 86, 5982 (2001).

[17] A. Altland, ptivate communication.

[18] S.Vishveshawara, T.Senthil, and M.P.A.Fisher, Phys. Rev. B61, 6966 (2000).

[19] Y.M.Blanter, Phys. Rev. B54, 12807 (1996).
[20] Y.Imry, Introduction to Mesoscopic Physics, Oxford Univ. Press (1997).

[21] T.W. Jing et al, Phys. Rev. Lett. 67, 761 (1991); A.G. Aronov and P. Woelfle, ibid 72, 2239 (1994); Phys. Rev. B50, 16574 (1994).

[22] A.V. Balatsky, M.I. Salkola, and A. Rosengren, Phys. Rev. B51, 15547 (1995); A.V. Balatsky and M.I. Salkola, Phys. Rev. Lett. 76, 2386 (1996).

[23] R. Bundschuh et al, Phys. Rev. B59, 4382 (1999).

[24] L. Taillefer et al, Phys. Rev. Lett. 79, 483 (1997); R. Movshovich et al, ibid 80, 1968 (1998); K. Krishana et al, ibid 82, 5108 (1999).

[25] H. Aubin et al, Phys. Rev. Lett. 82, 624 (1999). 